\title{
Ethische Fragen zur Technikanwendung im Kontext der Betreuung und Pflege alter Menschen
}

Heinz Rüegger

Der Einbezug moderner, computerbasierter Technologie in Pflege und Betreuung alter Menschen wird rasant zunehmen. Damit stellen sich neue Möglichkeiten, aber auch Gefahren. Der Artikel identifiziert ethisch sensible Bereiche solcher Technikanwendungen und nennt ethische Kriterien, die beim Einsatz entsprechender Technologien beachtet werden sollten.

Questions d'éthique concernant l'utilisation de la technologie dans le contexte de la prise en charge et les soins des personnes âgées

L'intégration de la technologie moderne et informatisée dans les soins et la prise en charge des personnes âgées va augmenter de façon fulgurante et apporter de nouvelles possibilités mais aussi des risques. Le présent article identifie les domaines éthiquement sensibles de ces applications technologiques et cite des critères éthiques qui doivent être pris en compte lors de l'utilisation des technologies correspondantes.

DOI 10.1024/2297-5160/a000008

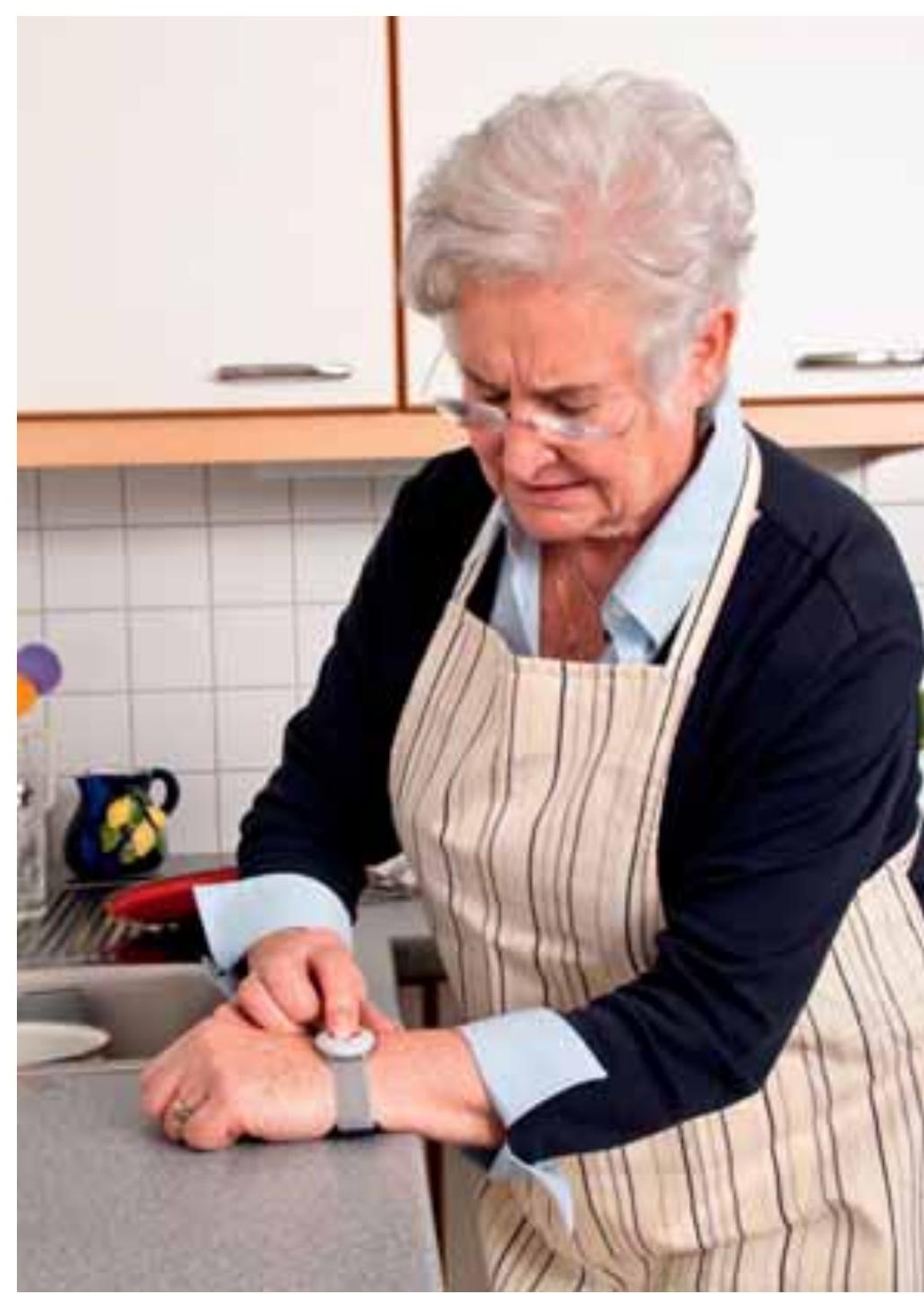

\section{Technik in der Pflege}

Der Einbezug technischer Mittel in Pflege und Betreuung alter Menschen gewinnt an Bedeutung. Das Forschungsund Entwicklungsfeld Assistierende Technologie (AT) in der Alterspflege steht vor einer rasanten Entwicklung. Im Fokus stehen dabei computerbasierte Technologien, die älteren Menschen helfen, ihren Alltag selbständig zu bewältigen, sie in ihrer Sicherheit und Gesundheit unterstützen, ihre Lebensqualität fördern und ihre Betreuung in Institutionen der Langzeitpflege erleichtern.

Schon heute hat die moderne Technologie in vielen Heimen ihren Platz im Alltag, etwa im Umgang mit elektronisch dokumentierten Daten oder bei Sicherheitssystemen (Alarm-, Notruf- und Ortungssysteme). Auch bei der Anwendung von emotional ansprechender Robotik wie bei der Spielzeugrobbe «Paro» kommt sie zum Tragen. Studien zeigen, dass ein erweiterter Einsatz von AT in Zukunft mithelfen könnte, den Mangel an Pflegefachkräften abzufedern (Becker et al., 2013). Es scheint heute unumgänglich, dass sich die Alterspflege ernsthaft mit diesen neuen Technologien auseinandersetzt. Dieses 
Thema ist nicht einzig unter technischen und ökonomischen Aspekten zu diskutieren, sondern muss ebenso soziale und ethische Gesichtspunkte einbeziehen (CURAVIVA Schweiz, 2014; Dorsten et al., 2009). Regierungsstellen in Deutschland und Österreich haben bereits eigene Studien zu ethischen Aspekten im Zusammenhang mit AT veranlasst (Manzeschke et al., 2013; Bioethikkommission, 2010).

\section{Die ethische Dimension}

Ethik hat mit grundlegenden Werten zu tun. Sie geht von der unveräusserlichen Würde aller Menschen aus. Jede Person hat das Recht, in ihrer Eigenart und Eigenständigkeit anerkannt, in ihren Rechten respektiert und bei Bedarf solidarisch unterstützt zu werden.

Im Blick auf AT besteht die ethische Dimension in der Klärung der Fragen, ob und wie die Technologien so eingesetzt werden können,

- dass sie den Bedürfnissen alter Menschen entsprechen und ihre Lebensqualität fördern;

- dass sie dem Anspruch auch des alten Menschen auf Selbstbestimmung, Freiheit, Privatheit und Sicherheit gerecht werden;

- dass sie die Arbeit der Betreuenden und Pflegenden unterstützen und erleichtern.

Es geht also um eine Orientierungshilfe für einen humanen, sozial verantwortlichen und professionell reflektierten Umgang mit den Chancen und Gefahren dieser neuen Technologien.

Dabei muss bei jeder Technikanwendung in Pflege und Betreuung klar sein, dass empathische Mitmenschlichkeit nicht durch unpersönliche Technik ersetzt werden darf: «Die Technik dient dem Menschen und sollte sich seinen Bedürfnissen, Wünschen und Lebensprozessen anpassen - nicht umgekehrt» (Manzeschke et al., 2013, 26). Studien haben gezeigt, dass die Akzeptanz gegenüber neuen AT in der Bevölkerung wie auch beim Pflegepersonal nicht zuletzt davon abhängt, dass die ethische Dimension ernsthaft berücksichtigt wird.

\section{Ethisch sensible Anwendungsbereiche}

Folgende Anwendungsbereiche neuerer Technologien in der Pflege und Betreuung werfen ethische Fragen auf:

- Datenerfassung/Dokumentation: Welche Personendaten sollen erfasst und gespeichert werden? Wer soll Zugriff auf diese Daten haben dürfen? Wie wird die Beachtung des Datenschutzes sichergestellt?

- Monitoring von Vitaldaten: Diskrete Sensoren können laufend immer mehr Vitaldaten von Personen (Blutdruck, Blutzucker, Atmungsparameter, Schlafrhythmus etc.) registrieren und auswerten, was gerade bei chronisch kranken Personen hilfreich sein kann. Wie aber vermeidet man die Gefahr der totalen Überwachung, die eine Person zum 〈gläsernen Patienten〉 macht und sie ihrer Privatsphäre beraubt?

- Elektronische Kommunikation: Moderne Technologie ermöglicht es, Kommunikation über fernbedienbare Bildschirme (Bildtafeln, open screens, individuelle Tablets) $\mathrm{zu}$ organisieren. Wie kann technisch vermittelte Kommunikation genutzt werden, ohne die persönliche Kommunikation, die gerade im Alter wichtig ist, zu vernachlässigen?

- Sicherheitssysteme: Viele Heime benützen bereits elektronische Bewegungsmelder, Notrufknöpfe, Ortungsgeräte, Überwachungskameras. Weglaufgefährdete, verwirrte Personen sollen geschützt werden und in Notsituationen rasch Hilfe erhalten. Wie können die sich zum Teil gegenseitig widersprechenden Werte - Sicherheit versus Privatheit und Freiheit - angemessen ausbalanciert werden?

- Technische Pflegeassistenz: Entwicklungen auf dem Gebiet der sozialen Robotik zielen auf die Herstellung technischer Geräte, welche die Pflege bei körperlich anstrengenden oder bei anspruchslosen Verrichtungen (heben/ wenden/mobilisieren von Personen, Post verteilen, Speisen/Getränke anliefern etc.) entlasten. Wie lassen sich mögliche Irritationen der zwischenmenschlichen Pflegebeziehung durch technologische Verfremdung vermeiden? Wie wird das Funktionieren der technischen Hilfen sichergestellt? Wer haftet bei Problemen?

- Virtuelle Realitäten simulierende technische Objekte: Heftige ethische Diskussionen haben sich um den Einsatz von Objekten mit interagierender Roboter-Technologie entzündet. So wirkt die Spielzeugrobbe «Paro» wie ein echtes Kuscheltier. Ist ihr Einsatz unverantwortliche Täuschung kognitiv eingeschränkter Menschen oder therapeutisch hilfreiche Anregung zur Kommunikation?

\section{Ethische Kriterien}

Will man die sich mit den Technikanwendungen stellenden ethischen Fragen klären, sind mehrere Prinzipien zentral:

- Benefizenz (wohl tun): Der Einsatz einer konkreten Technologie muss der betroffenen Person subjektiv wohl tun und darf ihr keinen Schaden zufügen.

- Selbstbestimmung: Technologie soll nicht gegen den Willen oder ohne das Wissen eines alten Menschen eingesetzt werden. Es gilt das in der Medizin- und Pflegeethik gültige Prinzip der informierten Zustimmung. Der Einsatz ist deshalb mit der betroffenen Person selbst oder bei deren Urteilsunfähigkeit mit der nach dem Erwachsenenschutzrecht stellvertretend entscheidungsberechtigten Person (Art. 378 ZGB) abzusprechen.

- Schutz der Privatsphäre: Jeder Mensch hat das Recht auf eine Privatsphäre, in der er vor den Blicken der Öffentlichkeit und der Kontrolle von aussen geschützt ist. Darum ist zu prüfen, wie viel Überwachung und fürsorgliche Kontrolle aus Sicherheitsgründen wünschbar ist und wo es die Würde einer Person verbietet, in ihre Pri- 
vatsphäre einzudringen. Sicherheit ist jedoch kein absoluter Wert. Ein bestimmtes Mass an Risiko gehört zum Leben. Darum sind Sicherheitssysteme, die die Bewegungsfreiheit einschränken, nur nach sorgfältiger Prüfung ihrer Verhältnismässigkeit einzusetzen. Angesichts des Rechts auf informationelle Selbstbestimmung ist die Erhebung von persönlichen Daten auf das Wichtige und Nötige zu beschränken. Gespeicherte Daten sind streng vertraulich zu behandeln.

- Transparenz: Ein transparenter Umgang mit elektronisch erfassten Daten und mit dem Einsatz technologischer Geräte ist zentral. Nur so kann eine Manipulation der betroffenen Menschen verhindert und ihnen ermöglicht werden, ihre informierte Zustimmung oder Ablehnung zu einer bestimmten Technikanwendung zu geben.

- Gerechtigkeit: Der Einsatz AT in der Pflege und Betreuung alter Menschen ist so zu gestalten, dass er allen Betreuten gleichermassen zu Gute kommt.

- Effektive Entlastung: Technikanwendung in Betreuung und Pflege muss die Betreuenden und Pflegenden tatsächlich entlasten und auch ihnen, nicht nur den alten Menschen, eine Erleichterung bringen.

- Ergänzung, nicht Ersatz: Der Einsatz von Technik in Pflege und Betreuung darf immer nur Ergänzung, nie Ersatz für persönliche Zuwendung sein: «Pflege entlasten, nicht ersetzen» (CURAVIVA Schweiz, 2014).

In der Praxis kommt es immer wieder zu Dilemma-Situationen, in denen ethische Werte oder moralische Prinzipien zueinander in Spannung geraten, z. B. Freiheit versus Sicherheit, Privatheit versus informierte Fürsorge. Doch es gibt keine simplen ethischen Standard-Lösungen. Gefor- dert ist ein situationsbezogenes Abwägen der Argumente, eine konkrete Assistierende Technologie zu nutzen oder darauf zu verzichten, und eine grösstmögliche Transparenz bei deren Einsatz.

\section{Literatur}

Becker, H. et al. (2013) Robotik in Betreuung und Gesundheitsversorgung (Zentrum für Technologiefolgen-Abschätzung TASWISS 58). Zürich:vdf Hochschulverlag.

Bioethikkommission beim Bundeskanzleramt (2010) Assistive Technologien. Ethische Aspekte der Entwicklung und des Einsatzes Assistiver Technologien. Wien (URL: https://www.bka. gv.at/DocView.axd?Cobld=39411).

CURAVIVA Schweiz (2014) Pflege entlasten, nicht ersetzen. Technische Assistenz in Alters- und Pflegeinstitutionen aus der Sicht des Pflege- und Betreuungspersonals. Bern (URL: http://upload. sitesystem.ch/7589311EBD/5D242FAD61/2CED7020C4.pdf).

Dorsten, A.-M. et al. (2009) Ethical Perspectives on Emerging Assistive Technologies: Insights from Focus Groups with Stakeholders in Long-Term Care Facilities. Journal of Empirical Research on Human Research Ethics, 4/1, 25-36.

Manzeschke, A. et al. (2013) Ergebnisse der Studie «Ethische Fragen im Bereich Altersgerechter Assistenzsysteme» (URL: http:// www.mtidw.de/service-und-termine/publikationen/ethischefragen-im-bereich-altersgerechter-assistenzsysteme/at_ download/pdf).

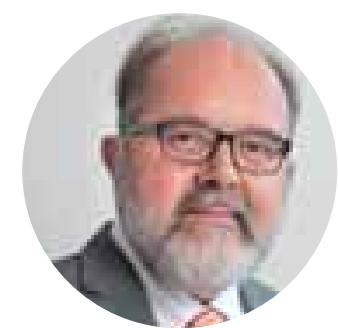

Dr. Heinz Rüegger, MAE ist Theologe, Ethiker und Gerontologe. Er arbeitet als wissenschaftlicher Mitarbeiter am Institut Neumünster, Zollikerberg.

heinz.rueegger@institutneumuenster.ch 